\title{
Lo sguardo in Africa di un giovane nefrologo
}

\author{
Lucia Palmieri \\ U.O.C. di Nefrologia e Dialisi, Policlinico Universitario di Modena, Modena
}

\begin{abstract}
A young Nephrologist in Africa
In a globalized world in which health should be a right for everyone, it is important to know the background of immigrant patients, in order to understand them and treat them in the best way. This article reports the 6-month experience of cooperation in Angola during the training in Nephrology within the project JPO (Junior Project Office) from the association "Doctors with Africa CUAMM". The one reported represents a very useful and enriching educational opportunity that allows acquiring many practical skills, gaining knowledge about several diseases, learning to work with the few resources available, making responsible choices in a difficult context of poverty and inequality, as well as working alongside with African staff.
\end{abstract}

Keywords: Cooperation, Training, Treatment choices

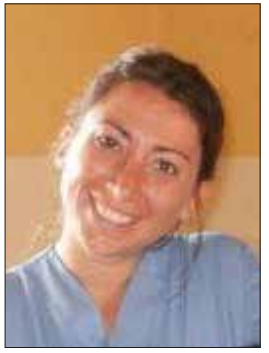

Lucia Palmieri

\section{Introduzione}

Sono un medico al quinto anno di specializzazione in Nefrologia presso l'Università di Modena. Da sempre sensibile alle problematiche sociali, alla salute dei cittadini come un diritto fondamentale e alla cooperazione internazionale. Durante i miei anni di studio universitari sono stata tra i fondatori della sede del Segretariato Italiano Studenti di Medicina (SISM) a Modena, un'associazione no profit nazionale creata da studenti di medicina che mira a migliorare la formazione degli studenti e a sensibilizzarli su tematiche quali la salute globale, con l'intento di coinvolgere gli studenti del mio ateneo nelle grosse tematiche sociali di interesse medico e nei processi di formazione di base, nonché di favorire lo svolgimento di fellowship internazionali presso ospedali esteri e di collaborare a progetti di cooperazione internazionale.

Ho aderito al progetto "Project for people" del SISM sia nel 2007 frequentando per un mese l'ospedale per donne e bambini dell'Indian Institute for Mother and Children di Calcutta,

Accepted: December 3, 2014

Published online: February 26, 2015

Indirizzo per la corrispondenza:

Dr. ssa Lucia Palmieri

UOC di Nefrologia e Dialisi

Policlinico Universitario di Modena

Via del Pozzo, 71

41124 Modena

lucia.palmieri@gmail.com sia nel 2009 presso la divisione di Pediatria e di Medicina Interna dell'ospedale di Wolisso fondato da Medici con l'Africa CUAMM in Etiopia. II CUAMM è un'associazione italiana che opera in Africa dal 1950 e si occupa di promozione e tutela della salute, rafforzamento dei servizi sanitari locali e formazione del personale locale, mediante l'invio di circa 1400 persone in 41 Paesi africani, prediligendo le località più povere e disagiate.

Durante il quarto anno di Specialità in Nefrologia sono stata selezionata per il JPO (Junior Project Office) dell'associazione Medici con l'Africa CUAMM, previa una lunga e attenta formazione che aveva l'obiettivo di fornire conoscenze specifiche, anche in termini di gestione sanitaria e di progettazione nell'ambito della cooperazione internazionale.

Ho compreso che è difficile fare una buona cooperazione in questi Paesi africani ed è necessario essere preparati e formati adeguatamente, cercando di comprendere la loro cultura locale e i mezzi a loro disposizione per lavorare realmente insieme "con l'Africa".

\section{L'ospedale di Chiulo}

Le mie attività si sono svolte presso l'ospedale di Chiulo nel sud dell'Angola nell'ambito dell'intervento di medici italiani a supporto dell'ospedale dal 19 settembre 2013 al 13 marzo 2014 (Figg. 1-3). L'associazione Medici con l'africa CUAMM ha garantito la supervisione della Dottoressa Paola Gaddi pneumologa ed esperta di medicina tropicale in qualità di tutor, il mio supporto logistico, l'alloggio, la copertura assicurativa e i permessi necessari.

L'ospedale di Chiulo, fondato nel 1954 dalle suore "Missionarie Mediche di Maria" irlandesi, è una delle due unità sanitarie di $2^{\circ}$ livello della provincia del Kunene. 


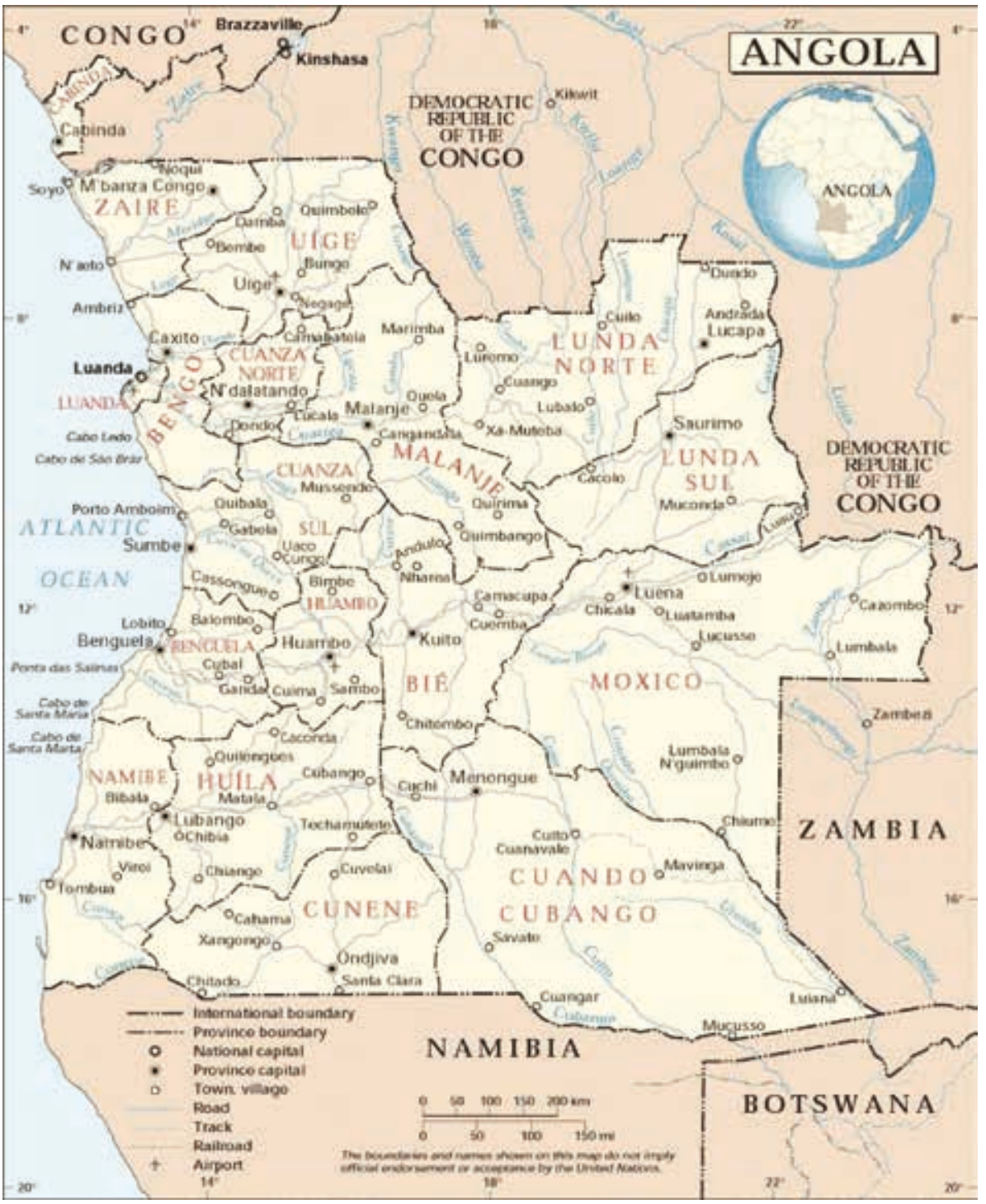

Fig. 1 - Cartina dell'Angola: Regione del Cunene al confine con la Namibia.

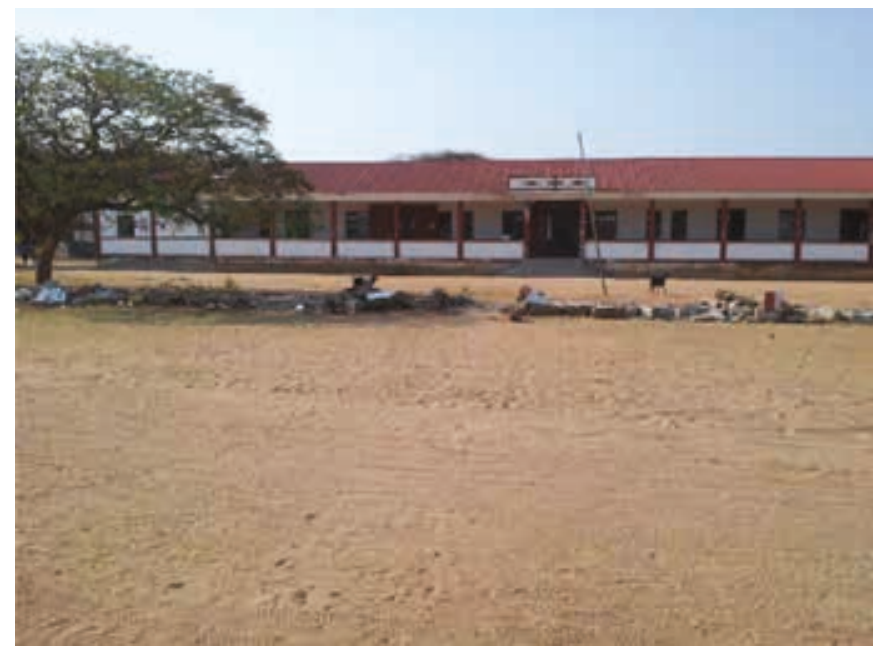

Fig. 2 - Ospedale di Chiulo: esterno.

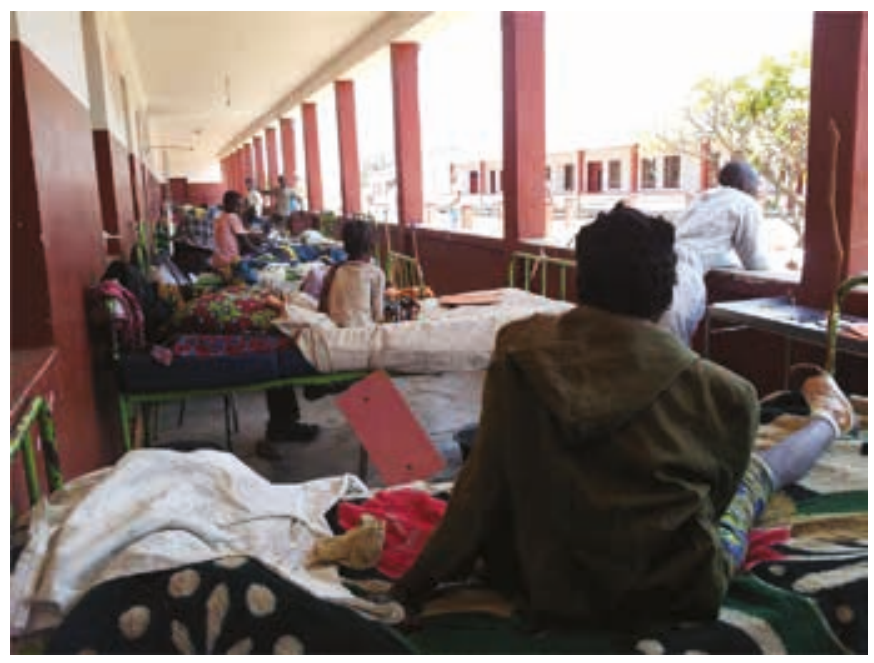

Fig. 3 - Ospedale di Chiulo: interno. 
L'ospedale è situato nella parte nord della provincia e raccoglie una bacino di utenza di circa 600.000 persone. Dispone di 250 posti letto ed è costituito da Pronto Soccorso, reparti di Pediatria, Medicina e Chirurgia, Maternità, Tisiologia, Laboratorio analisi, Radiologia, Sala operatoria, Farmacia e Salute Pubblica. Vi è inoltre una sezione carceraria con 10 posti letto per pazienti che devono effettuare trattamenti per tubercolosi, per patologie veneree o per malattie cardiovascolari. Annessa all'ospedale è la Scuola tecnica per Infermieri di base i cui dati dettagliati sono riportati nella Tabella I.

Di recente è stata aperta con successo anche un'area di dieci posti letto riservata a persone malnutrite con infermieri ed assistenti dedicati per la somministrazione del latte.

\section{L'esperienza in Africa}

Il team medico era composto da due colleghi CUAMM italiani, la mia tutor Dottoressa Paola Gaddi e suo marito pediatra Dottor Fabio Capello. Durante il mese di novembre 2013 fino a metà dicembre era presente anche il chirurgo e ostetrico Dottor Fabio Battisti esperto di ecografia. A questi da novembre si sono aggiunti dalla capitale anche due medici angolani neolaureati e una chirurga cubana molto esperta.

Il personale infermieristico di circa 80 persone era interamente angolano, come pure la direzione e l'amministrazione. Quando sono arrivata a settembre tutto l'ospedale gravava solo sui medici CUAMM e la mole di lavoro era immensa: in alcuni giorni dovevamo visitare fino a 80 bambini e avevamo contemporaneamente la gestione anche di 10 posti letto nella sezione intensiva per i bambini più gravi affetti da sepsi in corso di malnutrizione, malaria complicata (anemia, malaria cerebrale), meningiti, addome acuto (spesso a seguito della somministrazione di clisteri da parte di stregoni della medicina tradizionale), malnutrizione con altre complicanze, convulsioni febbrili, anemia falciforme complicata, febbre nel neonato, intossicazioni alcoliche.

Si lavorava intensamente tutti i giorni mentre le notti e i festivi eravamo reperibili in caso di emergenze (incidenti stradali, peggioramento improvviso delle condizioni di un ricoverato o parti cesarei).

Tre giorni a settimana era prevista l'attività ambulatoriale di tipo internistico rivolta a pazienti con le principali patologie croniche (ipertensione arteriosa, diabete mellito, insufficienza cardiaca, epilessia). Questo ambulatorio mi ha insegnato quanto sia difficile gestire le patologie croniche in Africa, a partire dal diverso concetto di tempo, poiché le persone sono abituate a gestire solo il presente e difficilmente riescono a pensare al futuro per cui il concetto di terapie preventive rimane spesso sconosciuto.

Da novembre alla fine di gennaio abbiamo dovuto gestire un'epidemia di colera (Fig. 4) isolando i pazienti contagiati in una sezione di un'ala dell'ospedale che era ancora in costruzione e in un tendone da campo.

Durante questo periodo ho acquisito una progressiva
TABELLA I - Ospedale di Chiulo

\begin{tabular}{ll}
\hline Bacino di utenza & 600.000 persone \\
Numero di posti letto & 250 \\
Reparti & Pediatria \\
& Medicina e Chirurgia Donne \\
& Medicina e Chirurgia Uomini \\
& Maternità \\
& HIV \\
Ambulatori & 3 Internistici e di follow-up dei \\
& pazienti dimessi \\
& 1 Chirurgico \\
& 1 Sala operatoria \\
Chirurgia & 60 posti letto \\
Tisiologia & 10 posti letto \\
Sezione carceraria & 10 posti letto \\
Sezione malnutriti &
\end{tabular}

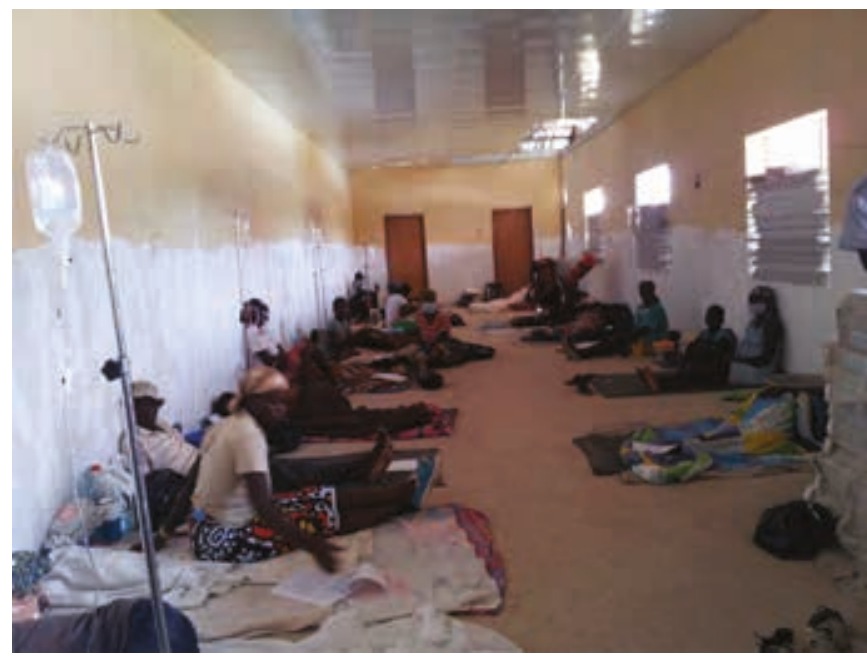

Fig. 4 - Emergenza colera.

autonomia nella conduzione del lavoro diagnostico terapeutico di routine in reparto di degenza medica per adulti e pediatrica, con particolare attenzione alla casistica internistica-infettivologica. Nello specifico negli ultimi mesi ero responsabile dell'intero reparto maschile da 50 posti letto e collaboravo con il Dottor Fabio Capello per la gestione della pediatria e del nuovo Centro malnutriti che avevo preso molto a cuore.

Ho dovuto imparare i protocolli angolani per la lotta all'HIV e alla tubercolosi; ho partecipato alla gestione di casi con HIV-AIDS. Purtroppo I'HIV si sta gradualmente diffondendo in queste zone e i pazienti portano uno stigma terribile, in particolare le donne vengono abbandonate dalle famiglie e spesso muoiono sole in condizioni disperate. 
Ho svolto numerose manovre diagnostiche non invasive: ecografie addominali, ecografie morfologiche in corso di gravidanza, studi ecografici di utero e annessi, ecocolor-Doppler vascolari per la diagnosi della trombosi venosa profonda, ecocardiogramma, ecografia toracica. Ho acquisito una graduale autonomia nell'esecuzione di manovre diagnostiche invasive nel paziente adulto. Inoltre come secondo chirurgo ho assistito a 35 interventi chirurgici tra cui laparotomie per perforazioni intestinali, cesarei, asportazioni di gravidanze ectopiche, amputazioni, rimozioni di osteosarcomi e raschiamenti.

\section{Un caso clinico speciale}

Clementina, una primipara di 18 anni incinta all'ottavo mese che non aveva mai fatto dei controlli sanitari durante la gravidanza, giunge in ospedale con ipertensione e convulsioni da eclampsia e viene subito trattata con idralazina, magnesio solfato e taglio cesareo in urgenza.

L'unico anestetico che avevamo a disposizione - la ketamina - contribuiva ad aumentare la pressione e nonostante gli sforzi, la madre continuava a convulsionare sul letto operatorio anche al momento dell'incisione. Dopo alcuni interminabili minuti finalmente riusciamo a salvare un bel bimbo di $3,7 \mathrm{~kg}$. Ho gestito il postoperatorio della madre con il prezioso aiuto delle ostetriche locali e dopo un risveglio complesso Clementina si è salvata e ha persino iniziato ad allattare il piccolo Fabio (Fig. 5). La madre e la nonna ci hanno ringraziato con un mare di "entu" che significa "grazie" nel dialetto locale.

\section{La formazione}

II CUAMM ha anche un importante ruolo di formazione del personale locale nell'ottica di evitare l'assistenzialismo puro e di "insegnare a pescare", favorendo in tal modo una futura autonomia dell'ospedale. Ho organizzato un corso sulla malaria rivolto ai laboratoristi, al personale medico (Fig. 6) e infermieristico.

Ho aiutato un collega a tenere un corso di sutura e di episiotomia su manichini appositi portati dall'Italia rivolto agli infermieri, che spesso si trovavano da soli a gestire ambulatori di prima assistenza nei villaggi isolati della savana.

Abbiamo tenuto un corso teorico-pratico di rianimazione neonatale rivolto a infermieri e ostetriche grazie alla mia esperienza maturata sul campo. È stato davvero emozionante poter rianimare e salvare quattro neonati sul tavolo operatorio mentre effettuavo come secondo operatore cesarei d'urgenza, si tratta di secondi interminabili in cui speri con tutto il cuore che la vita che hai tra le mani possa continuare.

Nel personale di Chiulo ho intravisto tanta voglia di imparare e buona volontà nonché una capacità di ingegnarsi con $\mathrm{i}$ pochi mezzi a disposizione: questi aspetti mi hanno riversato molta speranza per il futuro.

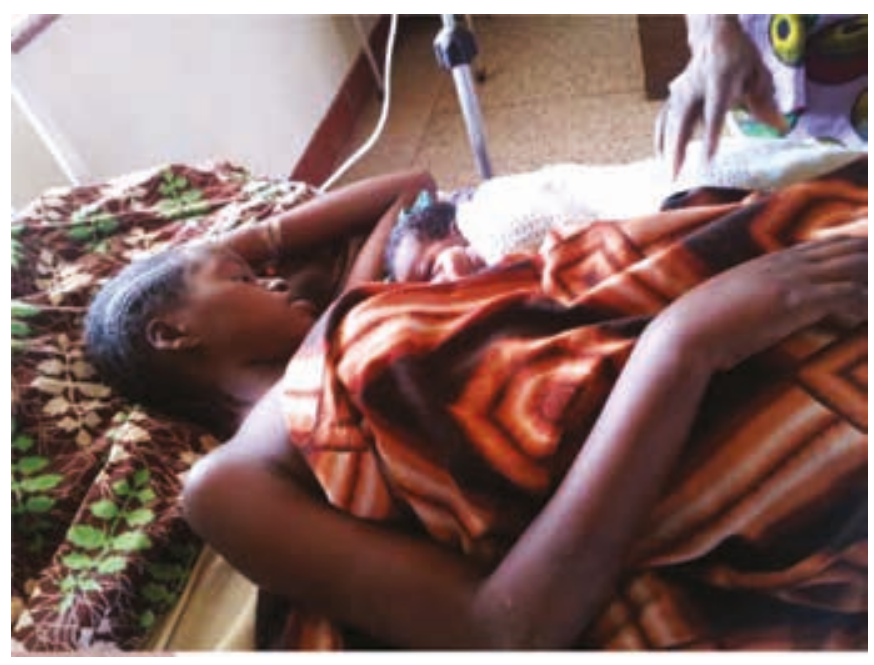

Fig. 5 - Clementina e il piccolo Fabio.

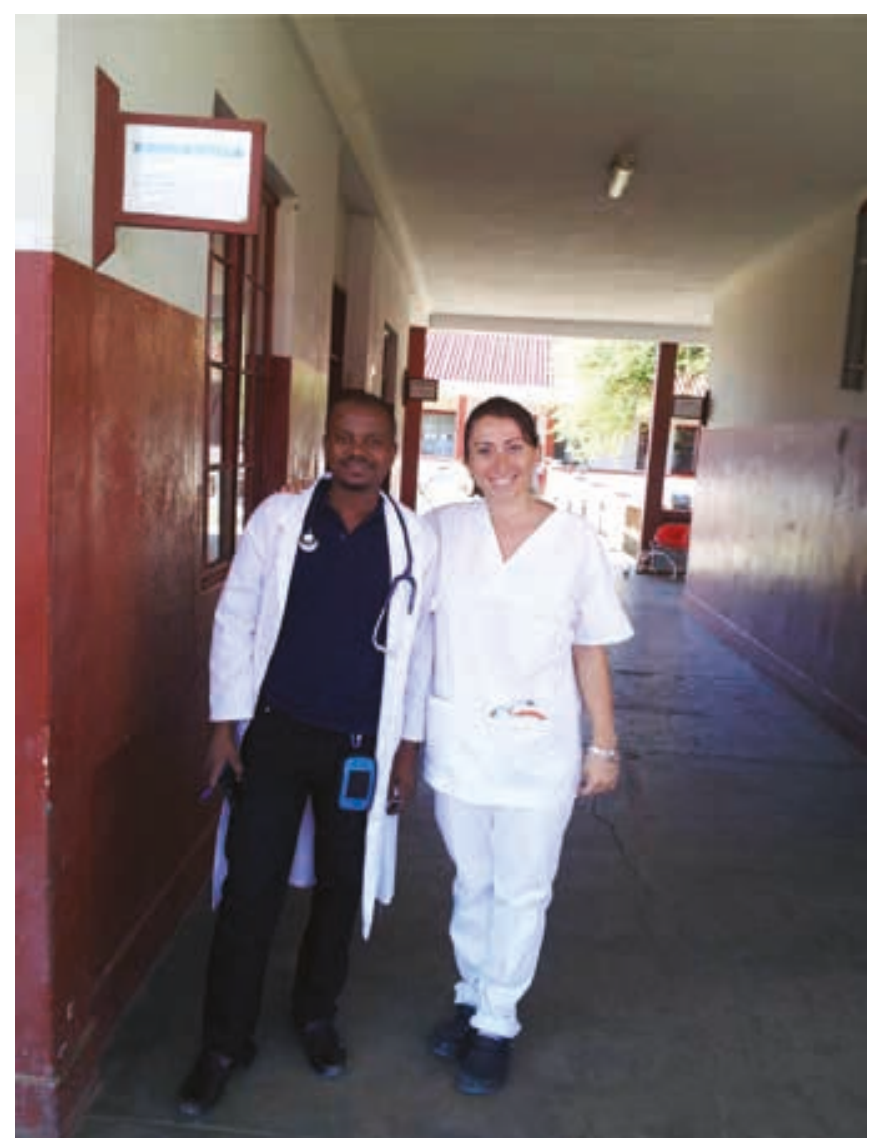

Fig. 6 - Medici con l'Africa: alla mia destra il Dottor Candida, giovane medico angolano.

\section{Conclusioni}

Questa esperienza mi ha molto arricchita umanamente e professionalmente, imparando a ragionare e a osservare i pazienti, a non dare mai nulla per scontato e a non sperperare 
le opportunità diagnostiche e terapeutiche che abbiamo in Italia. Inoltre il corso del CUAMM mi ha dato delle basi fondamentali anche per la gestione di un reparto, di una farmacia di un ospedale e di come affrontare un'epidemia. Ritengo che un'esperienza del genere possa essere di grande utilità anche nella nostra realtà, considerando il fenomeno crescente dell'immigrazione che ci porta di fronte a patologie e problematiche socio-culturali complesse e nuove. Capire la realtà da cui provengono i pazienti immigrati permette di potere comprendere e curare loro in modo migliore. Ho conosciuto persone nella povertà assoluta, grandi sofferenze e profonde ingiustizie. Ho capito come la salute sia, soprattutto in certi contesti, una questione di soldi, spesso nonostante tutto il nostro impegno, eravamo impotenti e nonostante facessimo diagnosi accurate mancavano mezzi, farmaci, specialisti e spesso i pazienti arrivavano troppo tardi in ospedale.

Penso che la mia scuola di Specialità in Nefrologia sia stata fondamentale nel mio percorso formativo poiché mi ha preparato adeguatamente anche di fronte a una realtà, quella africana, così complessa e difficile, dandomi anche buone basi internistiche, di infettivologia e di gestione di pazienti immunosoppressi che mi sono state utili nella gestione dei pazienti africani. Mi auguro che la Società Italiana di Nefrologia possa informare e favorire i giovani nefrologi che sono motivati a fare un'esperienza in contesti africani.

\section{Disclosures}

Financial support: No financial support was received for this submission.

Conflict of interest: The author has no conflict of interest. 